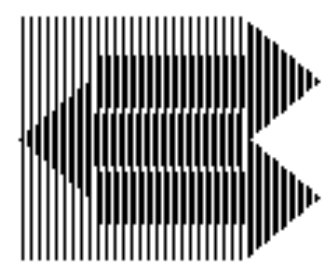

MIT EL 00-002

Energy Laboratory

Massachusetts Institute of Technology

\title{
Transmission Expansion in the New Environment
}

May 2000 


\title{
Transmission Expansion in the New Environment
}

\section{Yong T. Yoon and Marija D. Ilic}

Energy Laboratory Publication \# MIT EL 00-002

\author{
Energy Laboratory \\ Massachusetts Institute of Technology \\ Cambridge, Massachusetts 02139-4307
}

May 2000 


\title{
Transmission Expansion in the New Environment
}

\author{
Yong T. Yoon Marija D. Ilić \\ dreamer@mit.edu_ilic@mit.edu
}

Energy Laboratory

Massachusetts Institute of Technology

Cambridge, MA 02139

To Be Included As A Contributed Chapter In Power System Restructuring And Deregulation: Trading, Performance And Information Technology, edited by Dr. L. L. Lai, John Wiley \& Sons, Ltd., 2000 


\begin{abstract}
This chapter gives an account of the regulatory rules, rights and responsibilities essential for adequate transmission expansion in the new environment of the market-based electricity industry. We point out that the lack of incentives for transmission system expansion is the major cause for the current trend of declining reliability. The trend will continue unless the sufficient capital for the system expansion can be raised by the transmission owners/providers. The market mechanism needed for attracting such capital is presented in this chapter. It is important to recognize that in the new environment the role of transmission provider is no longer limited to managing physical transmission grids but also includes providing the communication infrastructure for transmitting accurate and transparent information regarding the system status to market participants in near real time. From the perspective of serving market participants as real customers through this new infrastructure the transmission provider is an active economic entity with the objective of profit maximization. With this in mind then the transmission expansion becomes merely a necessary ingredient for creating symbiotic relationship between the transmission provider and her customers. We conclude with describing a few indispensable tools in development for accommodating the evolving demand of customers in the market as presented in the chapter.
\end{abstract}




\section{INTRODUCTION}

At the initial stage of electricity restructuring in early 90's there were various reports estimating the expected improvements in efficiency with the introduction of competition. They range from the short-term effects; savings of $\$ 24$ billion to $\$ 80$ billion per year, or 10 percent of 40 percent off the average electric bill, to the long-term consequences; technological innovations and increase in reliability. Indeed the experience from the deregulation of telecommunication industry gave every indication that the similar benefits would be capitalized by simply dividing vertically integrated utilities into generation, transmission and distribution sectors and allowing competition to take place in generation sectors through divesture.

However, the reality of it is that the electricity restructuring process has been met with only few successes, far below the expectations, as well as with a couple of orders of magnitude more number of difficulties than that of telecommunication industry. Did people just expect too much? In order to answer this question, we must look into the assumptions that often follow with the introduction of competition.

The competition forces market participants to be more aware of their own profits. In simple economics terms, the profit consists of two parts: revenue and cost. From the supplier point of view, an increase in profit can be achieved either by decreasing costs or by increasing revenues. A decrease in costs is possible when the supplier can achieve higher efficiency from her existing plants, thus reducing the associated O\&M costs. An increase in revenues is possible when the supplier can expand her customer basis. ${ }^{1}$ From the consumer point of view, an increase in profit is directly related to finding a supplier who can offer the same quality goods at lower prices.

In the electricity industry the suppliers are the generators. Their costs constitute of various parts depending on the particular technology used to produce (electric) power; running a nuclear plant, for example, requires the incursion of (plutonium) fuel costs, O\&M costs, fixed costs, etc. Their revenues are the product of (electric) energy produced and corresponding electricity prices. The consumers, on the other hand, consist of distribution companies, electric cooperatives, market aggregators and in some instances, large industrial users. Their

\footnotetext{
${ }^{1}$ Throughout the chapter we assume no supplier has the market power so that raising her price to increase the revenue is not an alternative.
} 
costs are the electricity prices at which their loads are served.

In many parts of U.S. the energy market is structured in a way that there is no direct access between suppliers and consumers. As far as suppliers are concerned, their only customer is the transmission provider (TP), and for consumers, the TP plays the role of the sole supplier. This is due to the peculiar nature of electricity. Because there is no good practical means of storing electricity, the supply and the demand must be balanced continually. Plus, unlike in the telecommunication industry where a failure to execute a transaction results in "busy" signal, a failure to balance the system can result in system-wide blackout which can amount to astronomical figures in terms of losses. Therefore, the TP who is also the system operator must lead the coordinating effort in meeting the supply and demand with the scarce transmission capacity at times, and the easiest possible way to do so is by being in the middle and acting as the sole purchaser to suppliers and the sole seller to consumers.

Unfortunately in this market setup, the competition is always in a confined scope. In the short-run without the direct access which allows an active interaction between suppliers and consumers, there is a limit to how much suppliers are willing to lower the prices in order to expand their customer basis. More importantly, however, in the long-run no direct access means no customer choices, which is often the key to technological innovations. To make the matters worse, the market is structured so that in connecting suppliers and consumers, the TP does not assume any financial involvements due to her monopolistic stance. In order to overcome this dire situation, the current electricity market must undergo a little evolutionary steps so that there is a proliferation of direct access in the form of bilateral contracts.

Bilateral contracts are financial contracts written on the physical underlying of energy transfer involving only a subset of suppliers and consumers without the TP. ${ }^{2}$ As with other financial contracts, there are number of risks associated with bilateral contracts. The two major ones are the risks associated with future electricity prices and with transmission capacity. Because the participants enter into the contracts in advance, they are exposed to risk of future energy prices set by the TP on which the strike price is determined. This is, however, well understood in the world of finances, and there are many financial tools to deal with such risk. When the transmission capacity is scarce due to high level of demand, energy

\footnotetext{
${ }^{2}$ As a financial contract the bilateral contract needs not to be limited to physical transfer. However, for simplicity without loss of generality we consider only the contracts associated with physical transfer.
} 
transfers from certain parts of the transmission system to certain other parts are simply not possible or extremely uneconomical. Due to the high level of complexity in mapping financial bilateral deals with physical transfer, this risk is extremely hard to measure and has relatively few financial tools that can be complementary.

With the presence of bilateral contracts (and various other financial deals on transfer of electricity), the TP faces not only increase in operational difficulties with added complexity, but also a conundrum in planning as the market need changes far more rapidly than the transmission system can evolve. This has serious consequences in reliability as evidenced by recent system-wide blackouts. In the subsequent sections, we present a particular market structure that equips the TP with market-based solutions to conducting energy market with large quantity of bilateral transactions. This market structure also permits TP to become actively involved in market process despite the monopolistic stance. By allowing TP to pursue profit, it is shown that the transmission expansion problem can also be solved in an efficient way as intended with the introduction of competition.

\section{Role of Transmission Provider}

The electric transmission system is one of the most complex man-made systems. Due to the externality stemming from the operation of transmission system implementing market mechanism to the industry requires a fair level of understanding of not only economic, financial and regulatory aspects but also engineering consequences of restructuring.

Figure 1 shows the evolution of role of TP in the industry (as seen at the time of this writing).

In the dependent phase the TP functions as a part of vertically integrated utility.

In the passive phase the TP stands alone and oversees overall market activities. The market participants are required to submit their intended use of the system to the TP and based on that information the TP allocates transmission capacities following the strict rules set by regulators. The TP assumes no financial responsibilities and has minimal interactions with market participants. As shown in Figure 1 there are three different structures of TP under this phase.

In the active phase the TP participates in every phase of market activities. The functions by TP under this phase can be categorized into two: of market maker and of service provider. 


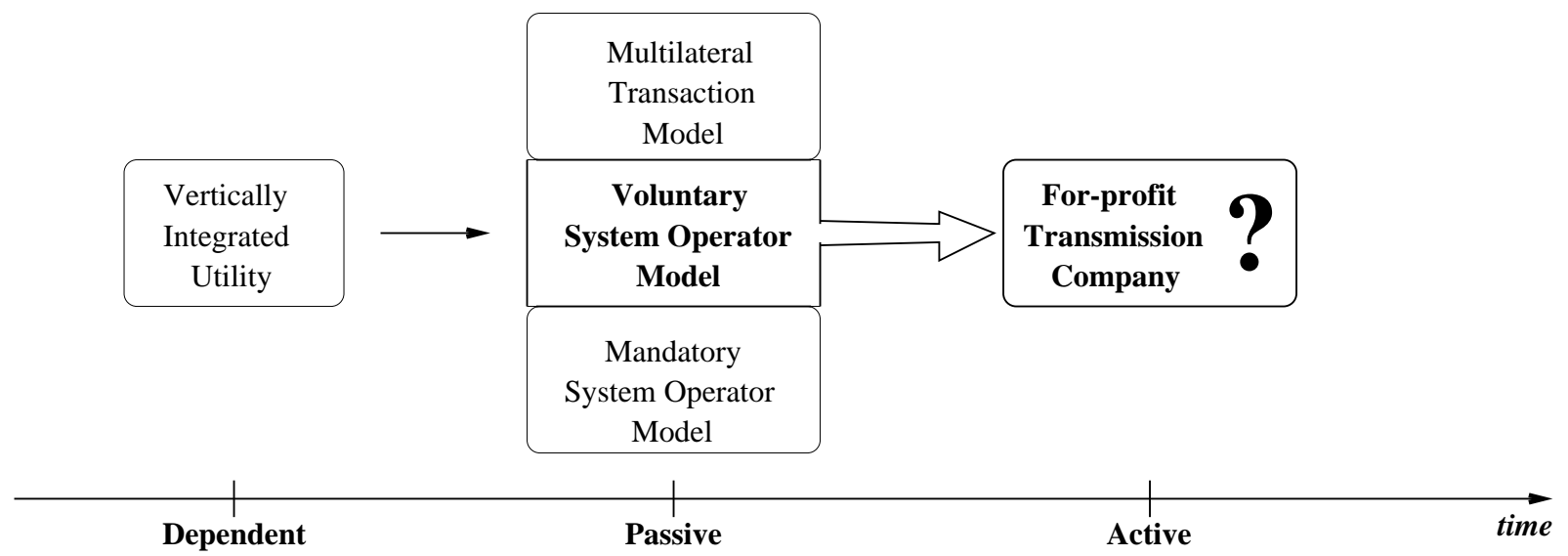

Fig. 1. Evolution of Role of Transmission Provider

Of these two only the function of market maker is under strict regulation. As a service provider the TP assumes full financial liability but is under no regulation.

We will discuss the role of the TP in each phase in details in the subsequent sections.

\section{A. Vertically Integrated Utility}

In the dependent phase the TP exists only as a part of a vertically integrated utility. The vertically integrated utility owns and operates a considerable amount of physical assets, including all of generating plants, transmission system and distribution networks over a sizable geographical area. The consumers in the area are the captive customers often referred to as "native load" and the utility is obligated to serve them under a strict regulation. In return, the utility is guaranteed to recover the cost of its prudent investment.

The operation and planning of the system by TP, therefore, can be viewed as a combined optimization problem of short-term generation scheduling and investment in new generation and transmission to balance load demand deviations ranging from hourly through seasonal and long-term and to do this at the lowest cost. A possible mathematical formulation of this problem is given as [4]:

$$
\begin{gathered}
\min _{I_{l}^{T}, I_{i, a}^{G}, P_{i, a}} \mathcal{E}\left\{\sum_{i} \int_{t_{0}}^{T} \mathrm{e}^{-\rho t}\left(c_{i, a}\left(t, P_{i, a}(t)\right)+C_{i, a}^{G}\left(K_{i}^{G}(t), I_{i, a}^{G}(t), t\right)\right) d t\right. \\
\left.+\sum_{l} \int_{t_{0}}^{T} \mathrm{e}^{-\rho t}\left(C_{l}^{T}\left(K_{l}^{T}(t), I_{l}^{T}(t), t\right)\right) d t\right\}
\end{gathered}
$$


subject to:

$$
\begin{gathered}
\frac{d K_{i}^{G}}{d t}=I_{i, a}^{G}(t) ; \quad K_{i}^{G}\left(t_{0}\right)=K_{i, t_{0}}^{G} \\
\frac{d K_{l}^{T}}{d t}=I_{l}^{T}(t) ; \quad K_{l}^{T}\left(t_{0}\right)=K_{l, t_{0}}^{T} \\
I_{i, a}^{G}(t) \leq 0, \quad I_{l}^{T}(t) \leq 0 \\
F_{l}\left(P_{G}(t), P_{L}\right) \leq F_{l}^{\max }\left(K_{l}\right): \quad \mu_{l}(t) \\
P_{i, a}(t) \leq K_{i}^{G} \quad: \quad \eta_{i}(t) \\
\sum_{i=1}^{n} P_{i, a}(t)=\sum_{j=1}^{n_{d}} P_{L_{j}}(t) \quad: \quad \lambda(t)
\end{gathered}
$$

where

$K_{i}^{G}$ : the amount of installed generation capacity at node $i$

$K_{l}^{T} \quad$ : the amount of installed transmission capacity for line $l$

$I_{i, a}^{G} \quad$ : the rate of investment in generation capacity using technology

$a$ at node $i$

$I_{l}^{T} \quad$ : the rate of investment in transmission capacity for line $l$

$C_{i, a}^{G}\left(K_{i}^{G}(t), I_{i, a}^{G}(t), t\right) \quad$ : the cost of investment using technology $a$ at node $i$

$C_{l}^{T}\left(K_{l}^{T}(t), I_{l}^{T}(t), t\right) \quad:$ the cost of investment in line $l$

$P_{i, a}(t)$ : the production using technology $a$ at node $i$, at time $t$;

$$
P_{G}(t)=\left[P_{1, a_{1}}(t) \cdots P_{n, a_{n}}(t)\right]
$$

$c_{i, a}$ : the cost of generation using technology $a$ at node $i$, excluding capacity costs

$P_{L_{j}}(t)$ : the uncertain (uncontrolled) load at node $j$, at time $t$;

$$
P_{L}(t)=\left[P_{1}(t) \cdots P_{n}(t)\right]
$$

$F_{l}\left(P_{G}(t), P_{L}\right) \quad$ : the flow on line $l$ as a function of system generation and demand

$F_{l}^{\max }\left(K_{l}\right)$ : the maximum allowable flow on line $l$ as a function of amount of installed transmission capacity; due to security constraints,

$$
F_{l} \ll K_{l}
$$

$\rho:$ discount rate of risk-free investment

$\mu_{l}(t), \eta_{i}, \lambda \quad$ : Lagrangian multipliers for corresponding constraints

The optimization period, $T$ in the problem (1) is the longer of two time intervals over which the generation or transmission investments are valued. As the system operator/planner 
decides the level of production and the rate of investment on generation and transmission, $P_{i, a}(t), I_{i, a}^{G}$, and $I_{l}^{T}$ serve as control variables in this formulation. The state variables of the system are $\mu_{l}(t), \eta_{i}(t), \lambda(t), K_{i}^{G}$ and $K_{l}^{T}$, for the status of the system operation can be accurately appreciated by examining these variables.

This formulation captures many well-known trade-offs relevant for the efficiency of the power industry; the relationship between the investment timing and the balance of the costs and benefits over time, the value of different technologies at different locations used to produce power, and complementarity of generation capacity and transmission capacity.

There are two noticeable features considering the operation and planning of the system by the TP (as a part of vertically integrated utility) as the combined optimization problem: the apparent complexity of the problem (1) and the implied assumption of return on investment based on costs $C_{i, a}^{G}, C_{l}^{T}$ and $c_{i, a}$. Due to the complexity, the solution to the problem is not readily available, and thus, the actual operation and planning of the system are performed suboptimally in many cases. Plus, since the rate of return on investment is determined based on costs, the optimality condition of the formulation is limited to concerning $I_{i, a}^{G}, I_{l}^{T}$, and $P_{i, a}(t)$. Nevertheless, the problem (1) is a valuable benchmark in studying the efficiency of the industry as the restructuring takes place.

\section{B. Three Models of Electricity Market}

In the passive phase the TP exists as a final authority in administering the market activities separate from generation and distribution sectors and indifferent from the financial consequences in a market environment. A newly created entity called, the system operator (or the grid operator), manages the system in order to insure the independence of the TP. The specific functions carried out by the TP are tailored to the market structure of the region she serves.

The structure of markets both existing and being developed is highly non-uniform. Depending on particular regional characteristics some markets admit centralized day-ahead and hour-ahead markets for wholesale trading and a real-time energy market for balancing while others only offer one or two centralized markets and still others offer only bilateral contracts among market participants with no centralized markets. Most of the markets in various regions within U.S. can be represented by one of three simplified market models: multilateral 
transaction model, mandatory system operator model and voluntary system operator model as shown in Figure 1.

The Multilateral Transaction model $^{3}$ is based on bilateral transactions among market participants. The model consists of three stages in completing transactions. First, individual buyers and sellers make bilateral trades with one another without disclosing the price and propose the agreed trades to the TP for physical implementation. The TP, upon receiving the proposed transactions, makes decisions whether or not to allow the transactions based on analysis of transmission network constraints. If the proposed transactions do not violate any constraints, then they are accepted without any modifications. This is the most desired case. If the proposed transactions result in violation of constraints, then the TP accepts none or a part of proposed transactions and suggests necessary modifications needed to the transactions in the form of public information called, "loading vector".[3] Based on this information, the market participants make new set of trades to satisfy the unmet demand while observing system limits.

Figure 2 shows the interaction among various market participants for the model. In this

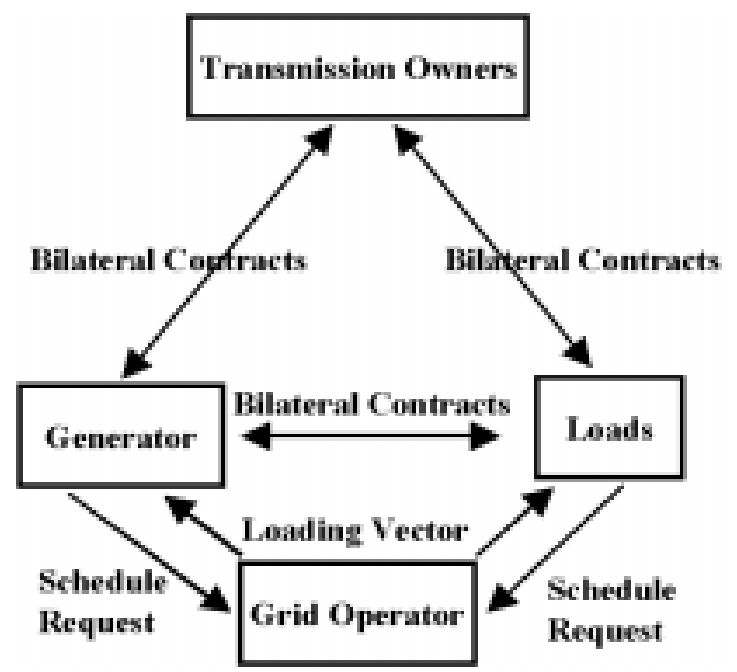

Fig. 2. Multilateral Transaction Model

model, the function of the TP is limited to verifying whether proposed transactions will result in violation of system limits.

\footnotetext{
${ }^{3}$ The proposed structure of Midwest ISO is closely related to multilateral transaction model.
} 
The Mandatory System Operator model ${ }^{4}$ is developed based on the existing practices by tight power pools. In this model the TP becomes the sole centralized market maker for overseeing economically and functionally bundled energy and transmission trades. The spot market refers to a place where this type of centralized market-based trades take place. Figure 3 shows the relation among market participants. Initially, market participants bid supply

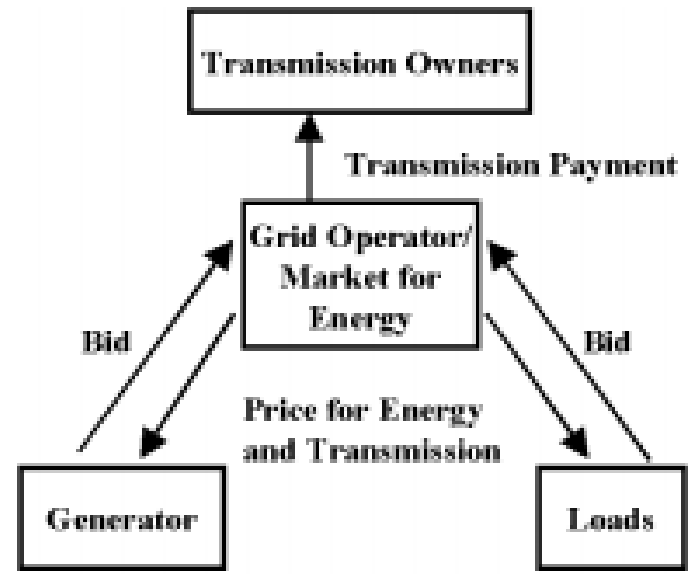

Fig. 3. Mandatory System Operator Model

curves $^{5}$ to the TP. The TP then simultaneously dispatches generators and allocates transmission capacity using optimal power flow program which determines the most economical mix of generations for given load.

The Voluntary System Operator model supports a multi-tiered structure that minimizes the TP's influence on profits by market participants while achieving acceptable level of reliability. Figure 4 shows the basic schematic of the model. In this model both bilateral and centralized market-based trades are allowed. The presence of spot market transactions is desired due to the requisite of continual balance of instantaneous supply with uncertain demand, particular to electricity industry while direct access and customer choice are achieved via bilateral trades.

The level of efficiency can be compared for each of the above three market structures by studying the operation and planning of system under each structure. Under the per-

${ }^{4}$ The structure of PJM ISO resembles the mandatory system operator model.

${ }^{5}$ Although a generalization can be made to include elastic demand in formulation, for the rest of the chapter we assume the consumers' demand is inelastic since not much is lost in term of the main purpose of the chapter. 


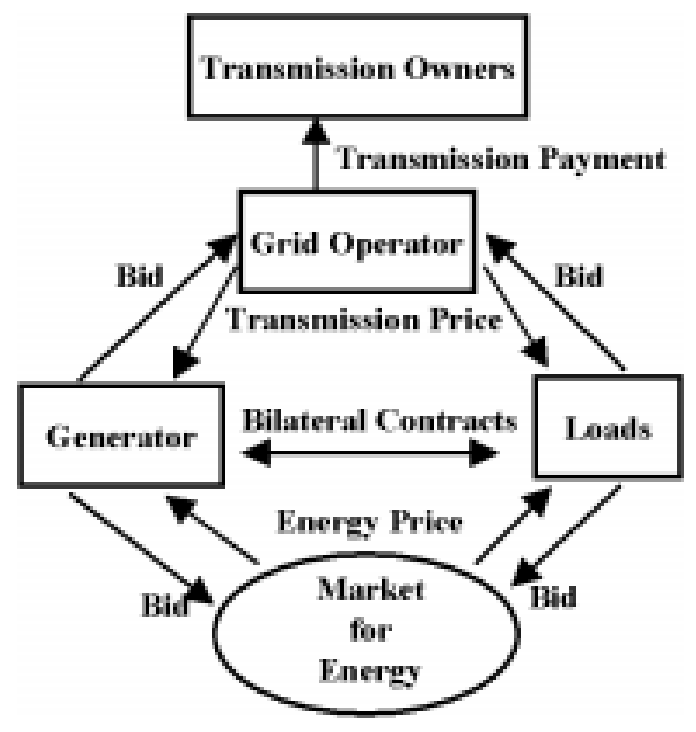

Fig. 4. Voluntary System Operator Model

fect market assumption with complete information, both the multilateral transaction model and the mandatory system operator model lead to an equilibrium solution of the following optimization problem:

$$
\min _{P_{i, a}} \mathcal{E}\left\{\sum_{i} \int_{t_{0}}^{T} \mathrm{e}^{-\rho t} c_{i, a}\left(t, P_{i, a}(t)\right) d t\right\}
$$

subject to

$$
\begin{array}{lll}
F_{l}\left(P_{G}(t), P_{L}\right) \leq F_{l}^{\max }\left(K_{l}\right): & \mu_{l}(t) \\
P_{i, a}(t) \leq K_{i}^{G} \quad: & \eta_{i}(t) \\
\sum_{i=1}^{n} P_{i, a}(t)=\sum_{j=1}^{n_{d}} P_{L_{j}}(t) \quad: & \lambda(t)
\end{array}
$$

The optimization defined in (8) is known as the (short-term) optimal power flow (OPF) problem. The result of solving the OPF problem yields

$$
p_{i}^{\star}(t)=\frac{d c_{i, a}}{d P_{i, a}}+\eta_{i}(t)=\lambda(t)-\left.\sum_{l} \mu_{l} \frac{\partial F_{l}}{\partial P_{i . a}}\right|_{P_{G}^{\star}}
$$

We make a couple of observations when comparing the optimization problems in (1) and (8). First, the formulation given in (8) is much more manageable than that in (1) due to the reduced complexity. This is the strength of market mechanism; by allowing the decentralized decision making, the overall system performance improves as the seemingly unattainable solution to the optimization problem becomes reachable. Second, the implicit 
assumption in the formulation in (8) is that $I_{i, a}^{G}$ and $I_{l}^{T}(t)$ are determined by individual suppliers and the TP respectively without considering the interaction between the two. This is the possible weakness of market mechanism; the expansion of the system could end up at a suboptimal state due to the lack of coordination between generation investment and transmission investment. It is very difficult (if not impossible) to formulate the planning aspect of the problem with Eq. (12) as the only coupling relation. As it stands, therefore, using either the multilateral transaction model or the mandatory system operator model and solving the optimization problem in (8) leads to a suboptimal solution different from that of (1) in most of the cases.

On the other hand, the flexibility inherent in the voluntary system operator model allows the formulation of another optimization problem needed to create another coupling relation between operation and planning. This problem is posed by defining the interaction of dynamics between bilateral transactions and the spot market. However, the TP must evolve into an active economic entity in the energy market before such problem can be posed.

\section{For-profit Transmission Provider}

Before discussing the additional restructuring steps needed in order to allow the TP to actively participate in market process, it is important to understand the underlying reasoning behind many regulatory rules insuring the independence of the TP from the generation sector and the distribution sector so that any regulatory change complies with the minimum requirement for independence based on the reasons given.

Unlike generation assets, the efficient operation of transmission system requires a single grid configuration rather than multiple grids serving customers in the same geographical region. This is due to the high degree of the economies of scale and the economies of scope related to transmission.

By having a transmission system covering a wide area of region, a large number of generators can be connected to a large number of customers. Under this configuration, the TP can serve the suppliers and consumers with the total transmission capacity smaller than the absolute sum of capacity demanded by individual transactions between suppliers and consumers. For example, this point is simple to illustrate through an example involving various transactions which commonly create counterflows on a single grid. Therefore, the 
$\mathrm{TP}$ as well as system users benefit from the economies of scale. In addition, by serving customers whose generation and consumption patterns differ from one another, a significant amount of system-wide savings can be achieved. For instance, it is easy to show that the total generation capacity required to serve various load is far smaller than the sum of peak consumption level of each load. Thus, the TP enjoys the economies of scope.

Given this monopolistic nature, a strict regulatory oversight is necessary when the TP takes on the role of a market maker. As a market maker, the TP allocates the transmission capacity by setting the price of energy and deciding which generating units to be dispatched. This is an important function of the TP especially at the time of scarcity in transmission capacity since this allocation process directly affects the profits of each market participants. It is not an overstatement that the success of deregulation of the industry depends on how well this process works so that the transmission capacity is distributed without resulting in distorted price signals and the overall electricity market achieves the highest efficiency level possible.

Because as a market maker the TP exerts a considerable influence on each participant's market activities, so much of time and effort has been spent in establishing the pricing for allocation of transmission capacity which will promote the efficient use of transmission and subsequently generation and consumption. The three market structures presented in the previous section all attempt to achieve this objective. The function of the TP as a market maker is, therefore, under strict regulation and will remain to be so regardless of any future regulatory changes.

The voluntary system operator model differs significantly from the other two models because the function of the TP is no longer limited to that of a market maker. In the multilateral transaction model the TP operates as a market maker by supplying the information necessary in accommodating bilateral trades. No explicit pricing setting by the TP takes place in this model. Therefore, the transmission revenue is strictly equal to the level designated through the rate of return regulation. In the mandatory system operator model, the TP sets the bundled energy and transmission price that minimizes that overall cost of satisfying the system load at each given instant. The transmission revenue in this model has two parts. The first cut is specified by computing the difference between the marginal prices assigned to consumers and to suppliers. The difference in the level of allowed investment recovery and 
the computed first cut is then compensated through approved access fees and usage charges assigned to consumers.

In the voluntary system operator model the TP is in a unique position to assign an explicit price for using transmission capacity due to the dual functions required for furnishing explicit bilateral transactions requested by a subset of market participants and for operating the spot market for the rest. The TP still assumes the role of a market maker in operating the spot market and is subject to strict regulation similar in the other two models. However, in allocating necessary transmission capacity for bilateral trades the TP functions as a service providers. As a service provider, the TP designs the appropriate transmission rates to be charged to each bilateral trade implemented without the regulatory oversight.

In implementing bilateral trades the TP conducts business just as any other for-profit entities; for given variable and fixed costs the TP functions in order to maximize her profit. We refer the for-profit TP to as independent transmission company (ITC).[1]

An increase in profit can be achieved either by increasing revenues and by decreasing costs. An increase in revenues is possible when the ITC can either raise the price for her service or expand the customer basis. Since the ITC can only set the transmission rates to be charged to bilateral trades while the spot market is operated under strict regulation, there is a clear ceiling to how much the price can be raised despite the monopolistic nature. On the contrary, the ITC has every incentives to lower the rate in order to expand the customer basis. Plus, related to the expansion of customer basis, the system reliability is expected to improve in order to attract more customers.

A decrease in costs is possible when the ITC can achieve higher efficiency from her existing system. This is directly related to intelligent handling of the limit $F_{l}^{\max }$ and flow $F_{l}\left(P_{G}(t), P_{L}\right)$ on each transmission line in Ineq. (5). For example, by improving the real time system coordination through better control design the ITC can effectively manage $F_{l}^{\max }(\mathbf{t})^{6}$ as the operating condition changes since the system is no longer required to operate at the conservative static $F_{l}^{\max }$ limits. The new technology such as flexible AC transmission system (FACTS) becomes also attractive for such devices allow a direct control of flows on individual transmission lines, $F_{l}\left(P_{G}(t), P_{L}\right)$.

The investment on transmission system expansion is expected to become more prudent ${ }^{6}$ We express $F_{l}^{\max }$ as a function of $t$ for it is no longer static. 
as well. Before committing to any major transmission project ITC is expected to spend a considerable amount of time and effort in analyzing the interaction between her investment and the decentralized decisions by participants on investment and production. Whether done analytically or through trial and error, the resulting effect of this type of pre-project activities is the understanding of complex relationship among $I_{l}^{T}, I_{i, a}^{G}$, and $P_{i, a}$ in the optimization problem (1).

Therefore, it is essential to transform TP into ITC by extending the function to include both a market maker and a service provider for achieving the short-run objectives of efficient system operation and the long-run objectives of prudent investment and technological innovations in introducing competition. An initial analysis shows that not only the market forces will eventually solve the optimization problem posed in (1) despite its apparent complexity, but also that the overall system can possibly out-perform the benchmark by incorporating $F_{l}^{\max }(\mathbf{t})$ and $F_{l}\left(P_{G}(t), P_{L}\right)$ as other control variables. ${ }^{7}$

In the subsequent section, we discuss the regulatory changes needed for transforming the TP into ITC.

\section{New Market Organization}

From the perspective of overall market development the transformation of the TP into the ITC is a consequential effect of an evolutionary process $^{8}$ of moving ahead with respect to the role of the TP from the passive phase to the active one. The ITC is assumed to be derived from the voluntary system operator model of the passive phase as illustrated in Figure 1. In the active phase of system operation the competition in the energy market is supported and promoted by the well functioning transmission ownership and operating structure of the ITC.

A few modifications to the regulatory policies become necessary so that the newly created ITC can serve as both a market maker and a service provider. It is important to recognize that any modifications to the policies must insure that the primary objective of achieving

\footnotetext{
${ }^{7}$ Before restructuring there is no incentives for the TP (or the vertically integrated utility) to include $F_{l}^{\max }(\mathbf{t})$ and $F_{l}\left(P_{G}(t), P_{L}\right)$ as possible other control variables in the optimization problem (1) since the return on investment is determined based only on costs $C_{i, a}^{G}, C_{l}^{T}$ and $c_{i, a}$ which are the direct functions of $I_{i, a}^{G}, I_{l}^{T}$ and $P_{i, a}(t)$ respectively.

${ }^{8}$ As described in the subsequent sections the system being operated by the ITC can admit a highly sophisticated market.
} 
greater market efficiency remains intact, by meeting the following minimum criteria:

1. Assuring open access: The ITC should remain operating as a common carrier.

2. Maintaining adequate reliability: The ITC should ensure the quality of transmission services to be acceptable by market participants.

3. Planning necessary transmission system expansion: The ITC should engage in transmission projects essential for meeting the changing need of market.

In accordance with spirit of free market, the market participants in all of generation, transmission and distribution sectors should partake in proposing the regulatory changes detailing the rights/incentives and responsibilities/penalties for the formation of the ITC. The role of the regulator should be limited to verifying whether the proposed changes meet the minimum criteria above while staying away from explicitly defining the functional requirements of the ITC.

The regulatory changes are directly related to the rate design applied to the ITC in allocating transmission capacity. The properly designed rate structure yields appropriate incentives for investment by the ITC. Equivalently, the ITC enters into the transmission project that reduces transmission system congestion only when the associated increase in social welfare equals the marginal cost of the investment. ${ }^{9}$ It is evident that the ITC must be prevented from benefiting from congestion under the rate structure.

Avoiding customer bypass is another direct benefit of properly designed rate structure. The customer bypass in the energy market is related to the uneconomical usage of transmission system. If the rate is set too low, a number of users are excluded from utilizing the system due to the inefficient allocation of transmission capacity. If the rate is set too high, some users choose to avoid using the system entirely. This customer bypass is an well understood phenomenon from the vertically integrated utility era. Under the top-down pricing the transmission tariff is set such that the avoided cost of generation is less than the total cost of distributed generation.

The rate design problem for the ITC can be approached from studying the development of rate structures in the era of the passive TP. In terms of the problem formulation the only difference between the passive TP and the ITC lies in the inclusion of long-run activities

\footnotetext{
${ }^{9}$ The investment is not restricted to the increase in transmission capacity $K_{l}^{T}(t)$ but includes the improvement in security limit $F_{l}^{\max }(t)$ and control of flow $F_{l}\left(P_{G}(t), P_{L}\right)$.
} 
of transmission system investment to the short-run function of efficient operation. The coupling of two separate time scale functions becomes feasible through considering the service provider aspect of the ITC in implementing bilateral trades. The incentives for the ITC to accommodating bilateral trades are created as a part of rate design problem so that the trades are implemented as long as the system configuration allows for a given operating condition. Prima facie the rate design incorporates the incentives for supporting the operating condition that grants the high degree of well balanced economic efficiency and technical reliability in short-run spot market just as required to the passive TP. Thus, the rate design problem is discussed from considering only the operation of spot market or equivalently the strict regulation required on the ITC in carrying out the role of a market maker.

\section{A. Incentive Rate Design - Price Cap Regulation}

The marginal pricing of transmission has been subject to extensive studies for the rate design assuming the passive TC. The main idea of the marginal pricing is the geographical differentiation in spot prices when a subset of transmission constraints are reached for a desired operating condition.[2] Under marginal pricing all significant network effects in operating the system are internalized in the resulting spot prices. Equivalently the resulting spot prices provide short-term signal for economic utilization of the system. This operating condition is also regarded to be most economical with respect to system-wide variable cost of generation.

The transmission rate design based on marginal pricing, however, is insufficient to generate enough revenues for the adequate recovery of investment on transmission system in supporting competitive energy markets. This is due to the inherent assumption in marginal pricing that the revenue is computed excluding the usage "rent" on uncongested transmission lines; the higher revenue results only with the higher level of congestion.

With the introduction of the ITC, the rate design based on marginal pricing is likely to lead to inefficient utilization of the transmission system. For example, by restricting transmission flows and thus raising the usage charges the ITC collects a higher net revenue. With this type of perverse incentives the ITC is expected to use all three means of restricting flows: manipulating generation dispatch, reducing transmission capacity through ill maintenance and delaying system expansion. 
With the transmission revenue from marginal pricing ranging typically around $25 \%$ of the total cost, the investment recovery problem requires a stipulation of a complementary charge in the rate design. The complementary charge is meant for recovering the fixed cost element of transmission investment without distorting short-run prices. This turns out to be a hard problem under general setting since no simple approach can meet the requirements of efficiency, objectivity and implementability. Some simplifying assumptions can be made if the consumers are restricted to having only the inelastic demand.

Under one approach to the ITC rate design the transmission charges have two components: market-based usage charges with some relations to the marginal pricing and access fees with the level agreed upon between the ITC and the regulator. The proper level of access fees should yield the ITC's revenue for recovering both fixed and variable costs with "appropriate" profits design to foster economic efficiency. The rate design thus includes a periodic review by the regulator of the ITC's profit and adjustment of access fees.

In order to create explicit incentives for promoting short- and long-run economic efficiency the price cap regulation is suggested on designing the ITC rate comprising market based usage charges and access fees. For price cap regulation the rate that can be charged by the ITC is capped by the price index. Although there is little experience with this type of incentive regulation for electric transmission in the U.S., the regulator can draw from the knowledge gained from gas industry.

In the gas industry it is found that by incorporating either $x$-factor or profit sharing the overall system efficiency improves despite the effect of uncertainty and the regulator's imperfect information. Both the $x$-factor and the profit share is a mechanism that passes a portion of benefits gained from cost reduction to the consumers, by defining the change in price index over a given period. In implementing $x$-factor the regulator and the ITC agree on the part of profit to be distributed back to the consumers on the ex ante basis whereas in applying the profit share is determining the portion on the ex post basis. When there is high degree of uncertainty in expected improvement in productivity, the ITC may prefer the profit share than $x$-factor since the profit share reduces the responsibility of the ITC. On the other hand, the flexibility bestowed by the $x$-factor may propel the ITC for higher increase in efficiency.

The practical application of price cap regulation requires a thoughtful consideration on the 
following issues:

1. Setting the initial rates: the initial rates are set by comparing the rate level ${ }^{10}$ to the total cost of the ITC including transmission losses, O\&M and fixed costs.

2. Revising the ongoing rates: the ongoing rates are periodically revised under regulatory review in order to ensure the proper performance incentives for each review period. It is important to recognize that the operating costs are not relevant during the period of the price cap since pricing is independent of cost once the rate level is determined.

3. Deciding the level of flexibility in rate structure: the rate structure ${ }^{11}$ is usually determined by the ITC in order to carry out necessary operational adjustment for improvement. However, this may lead to a significant volatility in pricing. The regulator may require to limit the volatility by placing restrictions on the cross subsidy under the price cap.

It is worthwhile at this point to investigate the practices used for computing the marketbased usage charges and the access fees related to the specific rate design especially setting the initial rates.

The market-based usage charges are commonly referred to as congestion charges. The nodal pricing and zonal pricing methods are two widely used methods in computing the congestion charges. The nodal pricing method compute the transmission rates by solving OPF given in (8). For a given time instant $t$, the problem can be solved by constructing Lagrangian function of the form

$$
\begin{aligned}
\mathcal{L}= & \sum_{i} c_{i, a}\left(P_{i, a}\right)+\lambda\left(\sum_{j} P_{L_{j}}-\sum_{i} P_{i, a}\right) \\
& +\sum_{l} \mu_{l}\left(\sum_{i} H_{l i} P_{i, a}+\sum_{j} H_{l L_{j}} P_{L_{j}}-F_{l}^{\text {max }}\right)+\sum_{i} \eta_{i}\left(P_{i, a}-K_{i}^{G}\right)
\end{aligned}
$$

where $\mu_{l} \neq 0$ if and only if $F_{l}=F_{l}^{\max }$. For simplicity, we use DC power flow in computing the flows on each line $H_{l i}$ in the system. The DC power flow equations in matrix notation are written as:

$$
\mathbf{B} \delta=\mathbf{P}_{\mathbf{G}}-\mathbf{P}_{\mathbf{L}}
$$

where $\delta$ is the voltage angle vector. Taking first derivative of $\mathcal{L}$ with respect to $P_{i, a}$ and

\footnotetext{
${ }^{10}$ The rate level refers to the expected revenue from the designed rate structure.

${ }^{11}$ The rate structure refers to the size of each component in computing price index.
} 
setting it equal to zero yields

$$
\frac{d c_{i, a}}{d P_{i, a}}+\eta_{i}=\lambda+\sum_{l} \mu_{l} H_{l i}
$$

Suppose the generation cost of supplier $i, c_{i, a}$, is a quadratic function of the output given by,

$$
c_{i, a}\left(P_{i, a}\right)=a_{i} P_{i, a}^{2}
$$

Then, under the perfectly competitive market condition, the optimal supply bid by supplier $i, b_{i, a}$, is the marginal cost bid given by,

$$
\begin{aligned}
b_{i, a} & =\frac{d c_{i, a}}{d P_{i, a}} \\
& =2 a_{i} P_{i, a}
\end{aligned}
$$

Matching the solution in Eq. (15) and the supply bid in Eq. (17), the system operator can set the price at node $i, \rho_{i}$, and the dispatch amount, $P_{i, a}$ as

$$
\rho_{i}=\lambda+\sum_{l} \mu_{l} H_{l i}
$$

Finally, the transmission rate is set by the difference in $\rho_{i}$ 's; i.e. $\rho_{i j}=\rho_{i}-\rho_{j}$.

The zonal pricing method consists of two steps: (i) aggregation of individual nodes into zones and (ii) computation of zonal prices. The system is first divided into a number of smaller markets by aggregating individual nodes into zones whenever there is little expectation of congestion within each market. The transmission rates is then computed by solving a similar optimization problem as given in (8); the cost $c_{i, a}\left(P_{i, a}\right)$ now represents the average cost of generation in zone $i$. The line flow constraints is now the congestion interface flow limit constraints, i.e., the power flow on any line $l$ along only the congestion interfaces is within the maximum rating of the line. The transmission rate is $\rho_{i j}=\rho_{i}-\rho_{j}$ where $i$ and $j$ now represent zones rather than nodes.

Although it may require higher sophistication in order to implement zonal pricing method from the ITC perspective, a significant reduction in computation complexity can be achieved in the rate design under the price cap regulation since only few number of zonal prices are needed to be considered rather than many nodal prices as is the case in nodal pricing. Plus, there is a greater advantage to be gained in implementing zonal pricing in accommodating bilateral trades as is illustrated in the subsequent section. 
The access fees are intended for recovering the fixed part of the ITC's costs and as thus is independent of actual usage. However, the usage independent charging for the access fees is impractical and may result in improper incentives for the ITC. In order to stipulate a meaningful charging mechanism, some measure of base load capacity needs to be given. A practical approach is to compute the access charges based on coincidental peak consumption of loads. The "12-CP" method [1] is one such approach. The portion of individual access fees is computed as

$$
S_{i}(t)=\frac{L_{i}(t)}{\sum_{i} L_{i}(t)}
$$

where $S_{i}(t)$ is the load $i$ 's share of system coincident peak, and $L_{i}(t)$ is the average of load in month $t$ at peak loading condition of each day. As the total revenue from this charge is equal to the product of access charge and the coincidental peak of each load, the approach provides the ITC with incentives to increase individual base load capacity.

Therefore, the price cap regulation and the rate design consisting the market-based transmission usage charges and regulator approved access fees offer the ITC an opportunity to recover its investment with some incentives for improvement in efficiency. However, the resulting rate structure does not immediately yield proper incentives for transmission expansion. In the subsequent section, a market mechanism called priority insurance service is discussed in terms of complementing the price cap regulation in order to provide right set of incentives for transmission system enhancement.

\section{B. Priority Insurance Scheme}

The driving forces of deregulation are aiming to establish a more competitive market in order to achieve lower rates for the consumers and higher efficiency for the suppliers. Through bilateral trades, the consumers can establish various service contracts with any supplier in order to obtain the lowest rate and most desirable service. Bilateral contracts specifying the amount of power, the time and duration of the service and the associated rate and possible compensation are negotiated and agreed upon between the suppliers and consumers. Since the proliferation of competition is directly related to the bilateral trades which allow direct access and customer choice, the success of market is dependent on the ITC's ability to administer the bilateral trades.

Since the transmission grid is a physical system, the ITC is able to honor and execute 
these bilateral contracts as far as the system design and operating conditions permit. Unlike in the spot market, the ITC is not allowed to participate directly in re-dispatching resources when executing these bilateral trades. Thus, the ITC relieves transmission system congestion through curtailing balanced bilateral trades or through creating counter-flows in the spot market by systematically adjusting rate structure. All bilateral contracts are required to specify the replacement resources in case of interruptions either due to the congestion curtailment or generator related contingencies.

Without loss of generality all bilateral contracts consist of the following specification:

$Q_{i j} \quad$ : the quantity of energy transfer

$i, z_{i}$ : the injection point and the corresponding zone

$j, z_{j}$ : the extraction point and the corresponding zone

$c_{i}^{G} \quad$ : penalty payment by generator $i$ for generator related contingencies determined ex ante

In case of curtailement it is assumed that the load is satisfied through the replacement generation purchased in the spot market of zone $z_{j}$ at zonal price of $\rho_{z_{j}}(t)$ paid by the load $j$. Otherwise, generator $i$ pays the transmission charge $\rho_{z_{j} z_{i}}(t)$ to the ITC.

Entering into a bilateral contract the supplier and the consumer face a few uncertainties related to transmission provision. Suppose the bilateral contract is signed over the period $t \in T=\left[t_{1}, t_{N}\right]$. Over the period $T$, any time the transaction is curtailed due to system congestion, the load is responsible for paying $\rho_{z_{j}}(t)$ which is determined ex post. Otherwise, the generator is obligated to pay $\rho_{z_{j} z_{i}}(t)$ which is also determined ex post. Thus, it is implicitly required that each time participants enter into bilateral contracts, they must estimate possibly highly volatile prices, $\rho_{z_{j}}(t)$ and $\rho_{z_{j} z_{i}}(t)$, and the probability of being curtailed $R_{i j}$ over the period $T$. This puts an extra burden to participants.

Priority insurance service offered by the ITC is designed to take away this extra burden. When the interested parties of bilateral contract request an allocation of transmission capacity for physical implementation of the trade, the ITC offers an alternative transmission pricing where they can agree on the transmission charge $p_{z_{j} z_{i}}$ determined ex ante for each time the trade is executed and the insurance payment paid to load $j$ by the ITC, $I_{z_{i} z_{j}}=\rho_{z_{j}}(t)-c_{i}^{T}$ where $c_{i}^{T}$ is equivalent to deductible payment. Plus, the system operator insures that the bilateral trade is interrupted no more than $x_{i j}$ times over the period T. By 
purchasing this service, the interested parties are completely protected from the volatility of $\rho_{z_{i}}(t)$ and $\rho_{z_{j}}(t)$.

In deciding to purchase the priority insurance, the interested parties must consider several factors, including:

- the opportunity cost of a curtailed transaction

- the probability of being curtailed with the upper bound given as $\frac{x_{i j}}{N}$

When the ITC provides priority insurance services to some interested parties, the transmission charges determined through the bottom-up approach since in essence the marginal valuation of transaction is reflected in $p_{z_{j} z_{i}}$.

In real time operation, the ITC determines the curtailment of bilateral trades needed for relieving transmission congestion along with computing spot market prices for each zone by optimizing her profit from both accommodating bilateral trades and conducting spot markets. Equivalently the priority insurance is implemented by solving an instantaneous minimum compensation problem over the entire network. Since there is a strict regulation on the ITC's role as a market maker, the profit from conducting spot markets is restricted. However, by imposing no regulation on priority insurance, the ITC may increase her profit as long as market can take. This creates an attractive incentive for transmission system expansion as a substantial effort by the ITC is expected in order to increase the customer basis for priority insurance service. The benevolence of this method is that because of the presence of spot market which is under strict regulation, the market's willingness to take the ITC's profit is well capped. Over time, the shortage (or excess) of customers in either spot market or bilateral trade is expected to level out depending on the ITC's ability to meet the changing need of market. The ITC with the system having better ability to adapt to market evolution is likely to have relatively higher level of customers subscribing to priority insurance service and to enjoy profits from business with no regulation.

In the subsequent section we discuss the effect of reduced regulatory uncertainty in revenue from providing priority insurance services on transmission expansion.

\section{Transmission Expansion}

The new market organization described in III above provides a fundamental setting for systematic transmission expansion. This task becomes an inherent part of the proposed ITC 
structure. A forward looking transmission provider actively learns the needs of its customers based on frequency and magnitude of congestion within its transmission system. Careful analysis of these data, together with the development of tools necessary for enhancing the system provides a basis for successful transmission enhancements and expansion. The timing of transmission enhancements and expansions in response to energy market needs becomes a very important aspect of the overall successful ITC business.

It is worthwhile observing that most of the immediate opportunities are likely to be for transmission enhancements by means of new control and information technologies. The prospects for new right-of-ways for new transmission lines are not as promising.

Another important aspect of dynamic transmission investments is the interaction with the regulators. The proposed ITC structure is Performance Base Rate (PBR)-based regulation dependent. It is not easy to design such regulation for networks, and it becomes necessary to develop right dialog between the ITC considering the details of their technical challenges, on one side, and the regulators, concerning the details of meaningful price cap design, on the other side, which is sufficiently flexible to take into consideration locational and temporal aspects of the electric power delivery. Only by having sufficient understanding of these details one could begin to harvest potential benefits which come from using control, communications and information at the right location and at the right time.

Possibly the most challenging part of transmission pricing for robust design is the need for price design and rates which are sufficiently simple and transparent to the users. The proposed market structure provides simplicity through effective zonal aggregation. This aggregation helps the users appreciate the needs for new transmission investments, internalize their value and, at the same time, actively develop secondary transmission market by exchanging transmission rights in near-real time. The transmission market liquidity is an essential part for successful implementation of ITCs. Therefore, the transparency provided on zonal level could serve as a fundamental catalyst of industry restructuring.

\section{Conclusions}

The development of new market tools for operating the transmission system becomes essential as the ITC moves into the active phase of management. In this phase the ITC is required to make complex business decisions over a wide range of time scales: long-term, 
short-term and near real time.

The long term decisions deal with the transmission system expansion. A fundamental question is related to computing the impact of the future demand on the system constraints and making system reinforcements in order to meet this demand. It is shown in the proposed transmission rate design that the investment cost is not directly affected by congestion rent at the spot market due to the high fixed cost element. Thus, no market tool for investment decision is required for the ITC based on spot market activities. Even when there is a significant congestion sustained over a long period of the time, the investment needed for relieving this congestion is a decision to be made by the regulator upon reviewing the performance of the ITC since the authority to modify the transmission rate lies on the regulator and not on the ITC. The activities in implementing bilateral trades and the implicated priority insurance services, on the other hand, is an immediate concern of the ITC in making the investment decisions. Typically the bilateral trades take place over an extended period and thus provide adequate revenue sources for investment recovery. The new market tools in long term project the demand in bilateral trades and in priority insurance services. The new market tools should make this projection based on the historical patterns on the users in subscribing into bilateral transactions sometime supported by priority insurance services, as well as the expected changes in customer basis. An investment into a new efficient generator by a participant is likely to follow by a request for implementing bilateral trades since such investment requires a steady flow of revenue. The better projection that the new market tools can produce, the more prudent investment the ITC makes and subsequently the higher earnings.

The short term decisions deal with pricing priority insurance services. This is perhaps the most difficult task by the ITC since the success of the ITC as an independent market entity depends on its ability to function as a insurance service provider.

There are three aspects to consider in the pricing. The first is refining the projection of bilateral trades from the long term market decision tool. Although only the aggregate volume is important in making investment decisions, the short-term decision requires an accurate projection of the locational and temporal patterns and the opportunity costs for each bilateral transaction. Over time, the market tool in this time scale can discover the patterns and the costs by extrapolating from previous seasonal behavior of the participants. 
The second is the valuation of insurance services given the specifications of a bilateral contract as described in Section III-B. The ITC has a menu of prices defined for different levels of reliability. In this formulation, the reliability is explicitly given in terms of the maximum number of interruptions $x_{i j}$ by the ITC over the contract period, $T$. This problem is similar to the option valuation and may be solved using similar market tools.

Finally, the third aspect of pricing is relating the decisions in providing the insurance services to spot market activities. Because the amount of the compensation depends on the deductible $c_{i}^{T}$ as well as the prices at the spot market, there is a high correlation between accepting bilateral trades and operating the spot market. The ITC is required, therefore, to solve for the optimal balance between bilateral trades and spot market transactions in terms of its profit. For instance, if the ITC deviates from this optimal and lean too much on the bilateral trades, there is an expected deterioration in the short term efficiency for which the ITC is responsible through the strict rate design. If the ITC, on the other hand, relies heavily on the spot market while neglecting the bilateral trades, the ITC may not be able to function as an active market entity. There are very few market tools available for solving this type of problem in other financial markets, but some active studies are underway.

The near real time decisions involve computing a combined optimization problem for minimizing insurance compensations to bilateral trades while maximizing the spot market throughput. These two are conflicting objectives and thus requires defining some offsetting weights when solving the combined optimization problem. The ITC can expand the conventional OPF tools as the new market tool needed for approaching the problem.

As the industry moves into the more mature stage of deregulation, the role of TP becomes more important. The new market tools described above are only the minimal changes required in the way the TP conducts its business as an active market participant, the ITC. It is, therefore, critical to build the tools that are consistent with the way they function over different time scales as well as with the other new business-oriented tools that are used by the participants.

\section{REFERENCES}

[1] Awerbuch, S., Hyman, L. Vesey, A. (Edited), Independent Transmission Companies: Unlocking the Benefits of Electricity Restructuring, 1999. 
[2] Schweppe, F.C., Caramanis, M.C., Tabors, R.D., Bohn, R.E., Spot Pricing of Electricity, Kluwer Academic Publishers, 1988.

[3] Wu, F.F., Varaiya, P., "Coordinated Multilateral Trades for Operation in a Competitive Open Access Environment", the Electricity Journal, March, 1996.

[4] Yu, C-N, Leotard, J-P, Ilić, M.D., "Dynamics of Transmission Provision in a Competitive Power Industry", J. Discrete Even Dynamic Systems: Theory and Applications, 351-388, Kluwer Publishign Company, 1999. 